\title{
LE VIE VERSO LA RAGIONE: \\ I SEGNi DEL NUOVO REALISMO IN GOMORRA ${ }^{1}$
}

\section{Torunn HaAland}

Abstract: Il saggio si concentra sui concetti di realtà e impegno etico e civile in Gomorra. Il romanzo-inchiesta di Roberto Saviano viene inserito nel contesto del ritorno al realismo e dell'emergere di "oggetti narrativi non-identificati” nella narrativa contemporanea, per poi essere associato al dibattito sul nuovo realismo sviluppatosi nell'ultimo decennio in campo filosofico. Costituiscono un punto di riferimento essenziale il Manifesto del nuovo realismo ed altri scritti di Maurizio Ferraris, così come La questione morale di Roberta de Monticelli. Il primo approfondisce la natura inemendabile della realtà ontologica, separandola nettamente dalla realtà epistemologica, mentre la seconda rivendica il valore della ragione pratica come base di conoscenza e giudizio. Entrambi i filosofi si oppongono allo scetticismo etico in favore di una rivalutazione della capacità della percezione d'accertare tutto ciò che non è socialmente costruito o che non è vero. Il fatto che ci sia una realtà esterna e indipendente dal sapere umano permette di formulare dei giudizi intesi a indirizzare l'agire umano. L'analisi di Gomorra mostra, precisamente, una tendenza a ricorrere ai sensi come prove dei fatti e alla ragione per acquisire una conoscenza approfondita dei fatti stessi. Le esperienze sensibili si trasmettono, inoltre, nella parola testimoniale e nella presenza fisica sul territorio dell'autore-testimone: due elementi chiave su cui si costruiscono le condanne delle realtà rivelate. I giudizi morali pronunciati suggeriscono che la libertà consiste nel dovere di portare a compimento i diritti civili e di rendere ragione e chiedere agli altri di rendere ragione del proprio agire. In fine, una tale concezione della scrittura e dell'impegno civile rivendica la capacità della parola scritta di documentare, come propone Ferraris, fatti e azioni, e la potenza della ragione quale fondamento per un dialogo critico con i lettori.

\footnotetext{
${ }^{1}$ Una versione preliminare di questa relazione è stata presentata al Convegno Internazionale dell'AATI tenutosi a Zurigo nel Maggio del 2014.
} 
Un decennio dopo l'esplosione del caso Gomorra, Saviano ci riporta alle circostanze e alle intenzioni che lo spinsero verso la stesura del celebre romanzo-inchiesta: "Volevo," egli spiega, "cambiare [...] una realtà che mi faceva schifo. Abbattere il potere di cui scrivevo e chiamare figuratamente alle armi, uniti, coloro che vi si opponevano" ("Gomorra, una storia"). Quando lo scrittore, oggi, si dichiara deluso e sconfortato dalle ideologie riformistiche come dalle istituzioni, esprime anche la consapevolezza che le aspirazioni rinnovative non si siano realizzate. L'insolito racconto di vicende mai così ostensibilmente esposte può aver raggiunto un pubblico transnazionale e tanti avranno accettato quel "nuovo patto" che sembra offrire, sentendosi coinvolti nelle realtà riportate e nelle denunce pronunciate. Nonostante l'entusiasmo che ha generato, però, l'esperienza letteraria non si è trasformata "in azione nel mondo" (Palumbo 220). Il mancato rinnovamento della vita locale e delle istituzioni nazionali sembra negare l'ipotesi di "incidere sulla carne del reale," mentre acquisisce nuovo valore l'ambizione umanistica di Vasilij Grossman di "salvare nell'uomo, quanto d'uomo ci sia" e ispirare quella "bontà" che lega il singolo al suo prossimo (Saviano, "Gomorra, una storia"). Si ridimensiona, perciò, la visione dell'impegno da parte dello scrittore ma non la fiducia che la scrittura, fittiva o non, possa promuovere la conoscenza della realtà. Tale convinzione si rimanifesta in modi diversi nelle opere successive di Saviano, ${ }^{2}$ come sembra rafforzarsi anche la consapevolezza di vivere in circostanze in cui "sapere, capire diviene una necessità. L'unica possibile per considerarsi ancora uomini degni di respirare" (Saviano, Gomorra 331)

La visione letteraria sperimentata in Gomorra parte dall'esigenza esistenziale di arrivare a una conoscenza approfondita di fatti accaduti e vissuti e si concentra sull'ideale di raggiungere una verità più complessa rispetto a quella che avrebbe potuto trasmettere una scrittura saggistica o giornalistica. Si uniscono, così, "il rigore della realtà e la suggestione della letteratura [...] la concretezza del dato e lo slancio della poesia" con il risultato che il reportage veritiero composto di dati non necessariamente documentati s'intreccia con ricordi soggettivi e con ricostruzioni spesso ai limiti del verosimile (Saviano, "Gomorra, una storia"). Tale strategia ha subito suscitato notevole e controverso interesse critico; alcuni avrebbero preferito

\footnotetext{
${ }^{2}$ Sia il romanzo-inchiesta dedicato al mercato della cocaina Zero, Zero Zero (Milano: Feltrinelli, 2013) sia il romanzo di formazione La paranza dei bambini (Milano: Feltrinelli, 2016) il quale narra infanzie rubate dalla Camorra, affermano il fondo sempre reale dell'impegno dello scrittore.

${ }^{3}$ Le citazioni successive saranno indicate con la forma abbreviata $G$.
} 
una non-fiction un po' più rigorosa o una fiction un po' più inventata (Policastro 187-188), altri hanno ritenuto che l'assenza di convenzioni narrative abbia accentuato favorevolmente quegli atti di parola che costituiscono la "forza agente" e la coerenza dell'opera (Benedetti 175-176,180). Deliberatamente inclassificabile e incentrato sull'io narrante, Gomorra è stato situato all'interno della letteratura di testimonianza (Inglese 59); non nel senso di "un'autofiction, bensì come un libro-azione" in cui l'autore, opponendosi alla realtà su cui indaga, agisce nel nome del lettore (Casadei 235), convinto che "l'esito etico della testimonianza" non dipenda da una veridicità oggettiva ma dalla garanzia che le parole enunciate e i fatti evocati "non saranno dimenticati" (Ricciardi 180).

Di fronte alla composizione eterodossa del libro si è spesso ricorsi al concetto d'ibridazione, suggerendo, ad esempio, che si tratti di "un'ibrida mistura" di concezione essenzialmente "etica" (Pocci 595). Altri propongono, invece, di andare oltre la "contaminazione" e la fusione di generi ben distinti che quest'operazione essenzialmente postmoderna sembra implicare per parlare, invece, di un "utilizzo di qualunque cosa possa servire allo scopo" di creare un testo fondamentalmente "epico" (Wu Ming 1). La prima interpretazione sottolinea la singolarità di Gomorra e il tentativo di "agire su chi legge" tramite le funzioni constatative e performative della parola (Pocci 596-598), mentre la nozione di un'epica composta da elementi storici o leggendari, da narrazioni ambiziose, e da problemi maestosi da risolvere, si riferisce a tutta una generazione - letteraria piuttosto che anagrafica - segnalata dall'invenzione di "oggetti narrativi non-identificati" (Wu Ming 1). Indifferentemente composti di elementi narrativi e saggistici; di tratti poetici e sguardi documentaristi, questi 'oggetti' possono cambiare la natura stessa del realismo, come dimostrato da Gomorra nella cui forma epica (Wu Ming 1) si può identificare l'unione tra una "rappresentazione mimetica lukàcsiana" e "lo straniamento brechtiano" (Gallippi 501-504). Il riavvicinamento alla realtà verificatosi nella letteratura contemporanea non presenterebbe, perciò, nessun recupero dei realismi passati, esprimerebbe piuttosto un "realismo riformato" nato dalla perdita dell'“egemonia" del postmoderno e dal ritorno della verità, della parola diretta e delle poetiche moderniste (Donnarumma 39-40, 54). Seguendo la centralità in queste opere della parola disornata e del "patto referenziale" stabilito col lettore (Inglese 61, 66), si potrebbe anche pensare a "un realismo tematico-referenziale" vicino, sia per le sue origini sia per l'aderenza al reale e la sua dimensione politico-civile, al mondo giornalistico (Palumbo 200-201). 
È precisamente sulla referenzialità della narrazione di Saviano che ci si propone d'indagare giacché a mediare fra le realtà osservate e un pubblico esteso ed eterogeneo sono proprio la concretezza del racconto e l'insistenza sulla verità dei fatti. Il senso critico e le premesse fenomenologiche intrinsechi a tale atto di resistenza avvicinano la scrittura di Saviano a certe tendenze di pensiero che proprio nel decennio di Gomorra hanno suscitato un proficuo dibattito filosofico. Si tratta delle correnti realiste emerse in opposizione alle concezioni scettiche del pensiero postmoderno e descritte con precisi intenti programmatici da Maurizio Ferraris nel Manifesto del nuovo realismo. In netta opposizione alla tesi nietzschiana secondo la quale il mondo vero è divenuto una favola ripetitiva dove soggetti sono solo le interpretazioni e non i fatti, il nuovo realismo rivendica la realtà e separa il sapere dall'essere per dimostrare che ciò che è esiste fuori dagli schemi concettuali (Ferraris, Manifesto 5). L'epistemologia dipende dalla costruzione sociale, mentre l'ontologia costituisce un'istanza inemendabile e non può, pertanto, essere soggetta a manipolazioni (Ferraris, Manifesto 63-66). Al contrario, la realtà resiste alle interpretazioni non fondate (Ferraris, "Esistere" 160-165) al punto da costituire uno "zoccolo duro" che, nella prospettiva del realismo minimale, o "negativo," proposto da Umberto Eco, segnala ciò che non è vero $(106,111)$. Secondo Ferraris, invece, si tratta di un realismo "positivo" nella misura in cui la durezza ontologica che denuncia il falso effettivamente conferma l'esistenza stessa di un mondo esterno. L'essere si registra, in particolar modo, tramite i sensi e se essi tendono ad avere l'effetto decostruttivo di smentire le nostre aspettative, le esperienze percettive accertano anche quella positività che ci permette "di stare al mondo" e d'interagire con gli oggetti in esso compresi (Ferraris, Realismo 11-15, 43, 69).

Dopo che le maggiori scuole di pensiero del secondo Novecento si sono concentrate sugli aspetti linguistici e sulla natura concettuale della realtà, con il nuovo realismo rientra nel campo filosofico la percezione - un aspetto dell'esperienza giudicato ingannevole dagli scettici ma che per Ferraris non ha nessuna funzione costruttiva (Realismo 35-42, 64). Al contrario, i sensi ci permettono di distinguere gli oggetti costruiti da quelli naturali per poi interpretare il mondo sociale e identificarne la relazione tra interpretazione e verità (Ferraris, Manifesto 64-69). Si raccoglie, perciò, il metodo decostruttivista ed ermeneutico caro ai postmoderni ma, inserita nei parametri programmatici del nuovo realismo, l'interpretazione non è fine in sé bensì mezzo per intervenire in maniera decisiva e con delle proposte concrete nel dibattito pubblico (Ferraris, Manifesto 71-5; De Caro e Ferraris 
vii-ix). L'intento di ricuperare il potenziale emancipatore della filosofia richiede, innanzitutto, di riunire la verità alla giustizia e il sapere alla felicità, eseguendo, in tal modo, un ricupero degli ideali illuministi che nella visione di Ferraris si rifa ad alcuni dei più autorevoli pensatori del secolo scorso. In primo luogo a Habermas, il quale già al momento del suo emergere avvertì gli aspetti anti-illuministi del postmoderno (Ferraris, Manifesto 32), ma anche a Lyotard, Foucault, e Derrida, i quali ebbero modo di riconsiderare le proprie teorie e di pensare un ritorno, rispettivamente, a Kant, alla parrasia praticata da Socrate, e all'Illuminismo quale via verso il futuro. Per Derrida, si trattava di rilanciare "l'idea dei Lumi" nel contesto contemporaneo e situarla "'nel progresso della ragione" (cit. in Ferraris, Manifesto 107), una proposta che si avvicina al progetto habermasiano orientato "all'egualitarismo della ragione kantiana" e alla visione di un agire integrato nella società; la razionalità comunicativa di Habermas non è "strumentale, funzionalistica e soggettiva," ma si diffonde nelle relazioni sociali e opera con strumenti critici e fini emancipatori (Brunkhorst 21-31). È in parte anche la concezione universalista della ragione quale parte dell'evoluzione più generale dell'umanità $\mathrm{e}$ perciò essenziale a qualsiasi impresa decostruttiva, che orienta il realismo positivo proposto da Ferraris. ${ }^{4}$

Che la ragione, lontana da essere assoluta, unica, immutabile, e tendenzialmente gerarchica, andrebbe rivalutata e riportata al centro del pensiero filosofico è precisamente quanto recentemente suggerito da Roberta De Monticelli. Intenta a collegare l'amoralità nella vita pubblica e privata degli ultimi decenni con la mancanza di un nesso a livello di pensiero tra "mores, politica e diritto," la filosofa dimostra che l'ascesa nel secolo scorso dello "scetticismo etico" ha provocato una "svendita" di quella "ragione pratica" sulla quale si costruì la modernità post-illuminista (13-16). Convinti, scetticamente, che non esista né verità di fronte ai giudizi di valore né, di conseguenza, oggettività rispetto ai giudizi intesi a indirizzare l'agire umano, ci si è allontanati da quel "pensiero pratico [...] che articola i giudizi di valore e ispira la produzione di norme" morali, giuridiche e politiche (De Monticelli 13-16). Stabilita questa premessa, il concetto della ragione si rileva alla luce della definizione socratica della "conoscenza:"

\footnotetext{
${ }^{4}$ A proposito dell'anti-illuminismo scettico, osserva Ferraris che "nel suo manifestarsi prima facie, quello della verità come puro potere è una affermazione molto rassegnata, quasi disperata: "la ragione del più forte è sempre la migliore" (Manifesto 96).
} 
opinione vera accompagnata da ragione. Opinione, cioè, che non è solo vera ma che ognuno può riconoscere per vera, in base alla ragione con cui viene giustificato il giudizio che l'afferma. (De Monticelli 91).

In termini concreti, la ragione fornisce una risposta alla domanda "'perché;"” un interrogativo in cui si materializzano le disposizioni universali di chiedere e di rendere ragione, e la risposta, ovvero, "la prova, l'evidence," in fin dei conti consiste nel vedere

con i propri occhi, e darmi la ragione e dirmi dove e come devo guardare per vedere che la proposizione affermata è vera. (De Monticelli 91)

Alla difesa della ragione s'intreccia, in questo modo, anche la rivalutazione della capacità dei sensi di accertare tutto ciò che è comunemente percettibile. Non a caso, le tesi avanzate da De Monticelli si legano ai principi più essenziali dell'Illuminismo - "autonomia, laicità, verità, umanità e universalită" — ed è con riferimenti a questi valori che il pensiero filosofico dovrebbe orientarsi verso una teoria della ragione perché si possa "fondare in filosofia, " ossia, in ragione, "la vita umana associata e quindi la civiltà e la cultura" (De Monticelli 96)

Il nesso che si ristabilisce tra ragione, verità, e conoscenza, da una parte, e la realtà a cui ci si avvicina con le percezioni e con gli intenti rinnovatori dall'altra, risuona in Gomorra il cui racconto s'ispira all'esigenza originaria di percepire e capire oggetti, ambienti, e relazioni velati dall'occultamento delle verità. Ci si rivela così un sistema nutrito dalla connivenza, dall'indifferenza e dalla paura, ma anche dalla diffusa sfiducia nella giustizia e nella possibilità che le cose possano cambiare. Il senso collettivo di vivere in circostanze incorreggibili non è smentito dalle vicende riportate e narrate da Saviano, ma in esse si riconosce, nondimeno, la convinzione che la consapevolezza dei fatti costituisca un fondamento morale e un'arma nella battaglia contro le realtà nascoste, distorte e negate dei potenti. La vocazione istintiva dell'osservatore narrante si trasforma, perciò, in quella "richiesta d'emancipazione" che emerge dal concetto socratico del valore morale del sapere e che si precisa nel discorso di Kant sull'Illuminismo (Ferraris, Manifesto 112). L'ideale di potersi valere "sicuramente e bene del proprio intelletto" e d'essere liberi d'esercitare, pubblicamente, la propria ragione (Kant 60-62) richiederebbe, secondo Ferraris, di separare kantianamente il reale dal non-reale per poi impegnarsi 
a modificare marxianamente ciò che è ingiusto (Ferraris, Manifesto 63-66). La composizione di Gomorra rivela una simile divisione d'intenti: il narratore distingue le realtà ontologiche da quelle immaginative e leggendarie, riportandole prima alle fonti primarie o alle esperienze percettive che le hanno scoperte ed esposte, e dopo al lettore stesso. Il ritratto che si crea non corrisponde certo ad alcuna forma oggettiva della verità — di essa si riconosce, al contrario, la natura essenzialmente "parziale" - piuttosto riflette un mondo pervaso da indiscutibili ingiustizie che diviene oggetto di precise valutazioni etiche fondate su un rapporto immediato e razionale con i fatti $(G$ 234). È così che l'atto di scrittura prende avvio dalla ragione, dalla presenza fisica, e dalla parola testimoniale e, oltre a denunciare la distruzione materiale e concettuale della realtà esposta, esso mira in ultima istanza a coinvolgere il lettore in un richiamo all'intransigenza e alla resistenza.

Il viaggio dell'enunciatore-autore comincia al porto di Napoli e si conclude nella Terra dei fuochi, zone simbolo di un territorio sfruttato fino allo stremo e di un'economia estesa a parametri globali. Nell'una, transitano 1.600 .000 tonnellate di merce cinese e "secondo l'Agenzia delle dogane [...], si calcolano duecento milioni di euro di tasse evase a semestre" ( $G$ 15); nell'altra, gli smaltatori della Camorra bruciano rifiuti tossici con gran vantaggio per le aziende settentrionali ed enormi danni per il territorio locale. Secondo "l'istituto superiore della sanità," infatti, "la mortalità per cancro in Campania [...] è aumentata negli ultimi anni del 21 per cento" ( $G$ 327). Riferimenti ai dati e alle loro fonti ricorrono nella descrizione di entrambe le zone, ma per capire i traffici illeciti nelle loro vere ramificazioni bisogna, spiega il narratore, "rifondare la propria immaginazione" ( $G 14)$, chiarificando così un aspetto metodologico di una scrittura che abbina impressioni e documenti ai fatti, attraverso l'immaginazione (Inglese 60). L'astrattezza statistica non renderebbe, per esempio, la distruzione di quello che era, una volta, l'incantevole paesaggio campano. Se, invece, si cerca d'immaginare l'infinità di navi che "s'immettono nel golfo $[\ldots]$ come cuccioli a mammelle" ( $G$ 12), si ricostruiscono anche i movimenti che hanno ingolfato il porto di terriccio, spazzatura e rimasugli al punto di farlo sembrare staccato dalla città come "un'appendice infetta;" "un anfibio" apparentemente non legato "né al mare né alla terra" ( $G 16)$. Allo stesso modo, l'entroterra campano ispira immagini mentali di "mappamondi della monezza" dove la terra, percorsa dal "sangue arterioso e venoso delle fabbriche" diventa un insieme di "cartine al tornasole della produzione industriale italiana” ( $G$ 313-314). Costruite di analogie suggestivamente concrete volte a evocare lo sfruttamento e le trasformazioni del territorio, queste 
visualizzazioni alludono all'orrore provocato da tali processi di degrado e servono perciò a coinvolgere il lettore e renderlo partecipe dell'indignazione di chi da anni li osserva.

Nel panorama di strategie comunicative adoperate, le informazioni colte e documentate s'intrecciano alle descrizioni fantasticanti e a ricordi e riflessioni radicati nell'intimità e nelle contradizioni che legano il testimone alle realtà sotto inchiesta. Le riflessioni e le denunce dell'autore dimostrano la volontà di condividere la conoscenza acquisita e provocare un senso d'indignazione nei lettori. Tuttavia, il bisogno quasi fisiologico di confrontarsi con il mondo criminale si manifesta, soprattutto, nei sensi che ne registrano gli oggetti e le forme. Sotto quest'aspetto si distingue, sicuramente, la partecipazione dell'autore a due attività lavorative simboli della modernizzazione e della graduale distruzione del territorio - lo scaricare merce clandestina al porto e l'impastare cemento nei cantieri, attività intraprese senza coscienza, nel primo caso e per sentire "fisicamente, nella budella," la potenza dei clan, nel secondo ( $G$ 231). Altre componenti dell'economia illegale, quali la produzione tessile ufficialmente inesistente nella provincia napoletana e le attività italiane ad Aberdeen dove vari boss riciclano redditi illeciti, richiedono una distaccata osservazione, a volte ripetuta e spesso facilitata da contatti personali.

Se l'attenta rassegna degli ambienti e delle storie umane presenta un invito a immedesimarsi nella posizione fisica di chi cerca le origini e le ramificazioni delle cose, l'attenzione prestata a visualizzare le vicende ritratte comunica anche il carattere essenzialmente percettivo delle esperienze. Il legame tra i sensi e la realtà si stabilisce in ambienti quali la piazza di spaccio inseguita precisamente per "l'alito del reale" che diffonde, un'aura di perpetua distruzione, calda e talmente vera che sembra permettere di "comprendere il fondo delle cose" ( $G$ 83). Come l'olfatto, anche lo sguardo è rivolto ad accertare realtà infuse di violenza per poi poter interpretarle e costruirne una conoscenza più profonda. Avvertito in piena notte del ritrovamento di un morto bruciato in una macchina, il narratore si reca a vedere il corpo reso irriconoscibile e deduce che il fuoco è servito a cancellare i segni della tortura per non provocare più del dovuto la rabbia e l'ostilità nel quartiere. L'osservatore onnipresente segue la vicenda in questura e, dopo ore di esami d'identificazione, resta sconvolto nel sentire che la vittima è Gelsomina Verde - una ventenne lontanissima dalle cerchie camorriste ma associata per una relazione passata a un traditore del clan. Mostratosi quest'ultimo irreperibile, gli assassini hanno colpito "nel modo più duro," per lasciare un messaggio 
inconfondibile ed evitare ulteriori tradimenti ( $G 96-7)$. L'enfasi posta sull'omicidio eseguito con ricercata premeditazione rivela l'intento di illustrare i meccanismi di una violenza che non distingue tra nemici e civili e che si mostra, invece, come mezzo di finezza feroce per dichiarare il proprio dominio sul territorio. A chi osserva con la determinazione di capire e di condividere la propria vicinanza e le proprie reazioni ai fatti, episodi come questo permettono nell'orrifica loro crudeltà di rendere percepibile una guerra che si svolge apparentemente all'insaputa del resto del mondo.

In ambienti dove omicidi e faide sembrano prassi del comune agire, non si fanno eccezioni per donne o innocenti, e sia la minuta contestualizzazione sia l'allusività grafica con cui vengono ricostruite le scene di violenza complicano ogni tentativo di distanziarsi dalle vicende raccontate e dalle riflessioni che ne evidenziano le conseguenze. L'importanza dell'incontro tra la percezione e la sfera ontologica in questo processo critico si manifesta emblematicamente di fronte alla villa sequestrata di Walter Schiavone. Nota nella zona come 'Hollywood' perché edificata sul modello della villa di Tony Montana - il mafioso cubano reso immortale da Al Pacino in Scarface - l'abitazione del boss arrestato costituisce un mito nella vita locale e il testimone ci si reca per "fissare" con i propri occhi i fondamenti sui cui si costruiscono e ricostruiscono le identità dei boss ( $G$ 268). La trasgressiva scelta d'introdursi nella proibita struttura ancora sorvegliata dai pali di Schiavone gli permette via via d'accertare come le leggende apparentemente esagerate su 'Hollywood' paiano "corrispondere al vero" e come nella fisicità della struttura si nasconda una fonte ideale per l'immaginazione (G 271-272). Quando la prospettiva dell'inchiesta si sposta dal mito alla realtà, l'incredulo visitatore si sarebbe potuto meravigliare di fronte a capitelli, strutture e scalinate rivelatisi identici a quelli dell'originale cinematografico. Egli, invece, consapevole di quante risorse e quante opportunità si siano sprecate per edificare questa villa così come tanti altri imperi al Nord d'Italia e in Europa, mentre nelle zone più sfruttate del Sud si continua ad accumulare rifiuti, s'indegna e in mancanza di altre strategie di protesta, decide di urinare nella formidabile vasca del boss. L'insolito gesto può sembrare insensato e certamente poco edificante ma sintetizza perfettamente il valore dei sensi nello sviluppo di una conoscenza che, in base ai fatti esposti, costruisce un messaggio di giustizia e libertà.

È da notare in questi episodi come la realtà possa decostruire le interpretazioni di chi la percepisce. Lidentificazione di Gelsomina Verde smentisce chi in un primo momento l'ha scambiata per uno di quelli che "si ammazzano tra di 
loro" (G 96-97) e 'Hollywood,' confermando le leggende, scredita i preconcetti dell'esperto osservatore. Questi casi concretizzano, con notevole precisione, la tesi che i sensi avvertono non solo l'inizio del processo cognitivo ma anche la resistenza dell'essere. Ferraris parla, a tal proposito, del "significato ontologico dell'estetica come l'aisthesis" la quale porta al realismo tramite esperienze di caratteri sorprendenti, differenti e aconcettuali, ossia non concettuali ("Esistere" 155-157). Ciò non vuol dire che il percorso del realismo, e la testimonianza che ne deriva, siano scevri d'incertezze. I sensi a volte ingannano, ed è il motivo per cui si distingue la realtà dalla verità. Davanti a realtà pluralistiche e sempre mutevoli poi, può essere che le percezioni, una volta trasformate in memoria, non trovino più riscontri. Nel regno della Camorra, però, ciò che più mette in questione l'attendibilità dei sensi è l'inverosimile ferocia dei fatti stessi. Per chi s'impegna a rintracciare le origini e i meccanismi del potere, le vicende inseguite e le loro circostanze appaiono inconfondibili; è "come se esistesse nel corpo qualcosa in grado di segnalarti la verità. Con tutti i sensi. Senza mediazioni” ( $G$ 151). Le problematicità presenti nella scelta di esporre fatti sistematicamente coperti emergono dopo perché non c’è più nessun pensiero

che possa attestare verità a ciò che hai visto. Dopo aver fissato una guerra di camorra nelle pupille, le immagini troppo numerose gonfiano la memoria [...] Non puoi fare affidamento sugli occhi. Non ci sono rovine di palazzi, dopo una guerra di camorra, e la segatura secca presto il sangue. Come se fossi stato soltanto tu a vedere o subire, come se qualcuno fosse pronto a indicarti col dito e dire 'non è vero'. $(G 151)$

È il terrore stesso del testimone: la cognizione o la coscienza di trovarsi solo e senza prove persistenti davanti a realtà non raccontate e spesso negate. Alla difficoltà di rendere credibili gli orrori più inimmaginabili si aggiunge l'eventuale incapacità o mancanza di volontà degli ascoltatori d'accettare per vero ciò che il testimone sa d'aver vissuto. Sarà anche per la consapevolezza d'aver trovato sia l'ispirazione sia il sostegno morale in un modello edificante che Saviano riconosce in Primo Levi la fonte principale della propria visione letteraria. Dopo aver letto Se questo è un uomo, egli spiega, citando al riguardo un prezioso commento di Philip Roth, "non puoi più dire di non esserci stato ad Auschwitz [...] sei lì e hai la certezza che la tua vita non possa più andare avanti senza metabolizzare quella 
esperienza." ${ }^{5}$ Che gli orrori vissuti e registrati si sarebbero mostrati tragicamente fugaci, i deportati lo avvertivano già nel Lager, dove le SS si divertirono a ricordare alle vittime che la storia delle loro sofferenze sarebbe sparita con loro, e che in ogni caso, se anche raccontata, nessuno l'avrebbe creduta possibile. L'esigenza di trasmettere una memoria si annunciava, inoltre, durante le notti insonni quando i prigionieri subivano l'angoscioso sogno di trovarsi a casa con persone care ma poco comprensive o semplicemente indifferenti verso le loro esperienze (Levi, I sommersi 11-12; Se questo è un uomo 53). Nel caso di Levi, tale incubo si realizzò perché il suo bisogno di raccontare si scontrò con il desiderio collettivo di dimenticare i fatti del Nazi-fascismo (Levi, "Appendice" 157). Avrebbe potuto chiudersi nel silenzio il superstite della Shoah, ma era inutile chiedersi se non vedere, e non esserci forse sarebbe stato meglio. Saviano, invece, ha scelto d'opporsi al silenzio e alla cecità dell'indifferenza collettiva per confrontarsi con un potere criminale per tanti versi anch'esso totalitario. Ciò che in particolar modo accomuna le loro testimonianze è l'impegno di denunciare non solo l'annullamento sistematico di ogni segno di resistenza o opposizione collettiva, ma anche le strategie adoperate per nascondere o distorcere la natura stessa delle strutture violente e screditare chi si propone di raccontarne la verità.

Non a caso è proprio con riferimento alla possibilità teorica di negare ciò che è o che è stato che Ferraris respinge la proposta scettica di prendere congedo dalla verità. ${ }^{6}$ Seguendo le conseguenze estreme della tesi che non esistano fatti e che la realtà sia riducibile a interpretazioni, si potrebbe, in effetti, sostenere che i Lager non siano esistiti, o che la Camorra sia una fantasia. Chi, invece, afferma l'aconcettualità degli eventi storici e la resistenza dell'essere riconosce nel realismo il fondamento etico di un impegno percettivo e analitico inteso a disassociare la realtà dalle interpretazioni falsificanti (Ferraris, Manifesto 21-23; Ferraris, "Esistere" 163). Oltre al fatto che non è possibile costruire un progetto liberatorio sulle teorie scettiche, è essenziale riconoscere che le ingiustizie presenti e passate richiedano un'azione critica capace di riconnettere il sapere e l'emancipazione al fine di tutelare la giustizia e chi nella società si trova escluso e soppresso (Ferraris, Manifesto 62). Se si accetta l'esistenza non solo di una sfera esterna che ci obbliga a vedere e a sentire, ma anche di un libero arbitrio che ci permetta di agire, risulta

\footnotetext{
${ }^{5}$ Ricordiamo che l'audiolibro pubblicato per Emons nel 2013 di Se questo è un uomo presenta la lettura di Roberto Saviano (Saviano, "Saviano legge").

${ }^{6}$ Va notato che il realismo filosofico di cui si fa portavoce Ferraris appare in esplicita replica all'Addio alla verità (2009) di Gianni Vattimo (si veda Ferraris, Manifesto 92-97).
} 
più chiaro come i sensi e la ragione servano a indirizzare le azioni verso fini morali. Fondata sulla distinzione tra sapere e potere e tra essere e interpretazioni da un lato, e sulla convinzione, da un altro, che la filosofia non sia fine a se stessa ma che debba cercare un coinvolgimento con la realtà, il nuovo realismo ci offre una preziosa chiave di lettura non solo per le rivendicazioni di verità espresse in Gomorra ma anche, e soprattutto, per i principi etici su cui si fonda la visione dell'autore.

Fino a che punto la fiducia nel dovere di affrontare la realtà con i sensi e con le parole costituisca un fondamento etico e metodico di Gomorra si capisce dall'elogio espresso a coloro che prima di Saviano si sono opposti al potere criminale. Pensiamo all'assessore Antonio Cangiano, rimasto paralizzato dopo un attacco dei Casalesi, e al Senatore Lorenzo Diana il quale, dopo indagini spesso ostacolate e sempre ricominciate, si è costruito un'arma di conoscenza e di memoria ( $G$ 223). Come loro, anche l'ex-sindaco comunista di Napoli, Renato Natale, spaventa i clan, e la protezione metafisica che lo circonda aumenta proporzionalmente con il coraggio e la costanza ( $G$ 252). L'integrità intrinseca dell'attività di questi politici trova il suo emblema nell'ardire di una giovane maestra: Trovatasi testimone di un assassino, non ha scelto, come le ragazze con lei presenti, d'abbassare lo sguardo, ma ha identificato e denunciato l'agguato, permettendo la condanna all'ergastolo dell'assassino, con la conseguenza però di vedersi costretta a lasciare città, casa e lavoro. Lo scandalo provocato dall'insolito gesto di giustizia non riflette, però, tanto la denuncia di per sé bensì la

scelta di considerare naturale, istintivo, vitale poter testimoniare. Possedere questa condotta di vita è come credere realmente che la verità possa esistere, e questo in una terra dove [...] la verità è sempre la versione dei potenti, dove viene declinata raramente e pronunciata come merce rara da barattare per qualche profitto. $(G 307,306)$

A esporre non solo i delitti ma anche le falsificazioni e i negazionismi al servizio del potere si era dedicato anche don Peppino, il quale si propose di affrontare le realtà terrestri, dall'altare come nelle strade, rifiutandosi innanzitutto di assecondare la presunta fede dei boss e di contenere la loro attività "nel silenzio confessionale" ( $G$ 245). La sua battaglia fatale si nutriva dell'esigenza di andare oltre le verità teologiche per accertare la realtà ontologica e identificare in essa la ragione d'essere di una missione morale indirizzata non verso l'al di là, ma verso la costruzione di una civiltà migliore. 
Nell'attività e nelle attitudini di questi cittadini esemplari si riconoscono soprattutto tre valori civili che nel racconto di Saviano s'intrecciano con la ricerca risoluta dei fatti e con l'atto stesso dell'enunciazione: la potenza della parola, la prossimità alle vicende raccontate e la ricerca dei fondamenti più concreti della Storia. Emergono, in primo luogo, gli enunciati che nella lotta per la verità si scontrano con un tacere nato dall'indifferenza ancor prima che dalla paura. Che gli enunciati pronunciati in difesa della legalità e della civiltà rappresentino una minaccia agli occhi dei clan non dipende solo da quanto rivelano, ma anche dalla loro capacità di raggiungere cerchie sempre più estese di lettori e di nuovi narratori. Come le testimonianze della maestra e di don Peppino, combattute mediante l'ostracismo e l'assassinio, Gomorra si rifá con termini fortemente referenziali a una vita locale riconosciuta e nota come quella dell'enunciatore-autore. Ne espone un sistema di abusi e violenze, ne prende le distanze e intanto attraverso le sue denunce e riflessioni mira a coinvolgere gli indignati e svegliare i sonnolenti. In questo senso, il racconto acquisisce una "forza agente" composta, come dimostra Carla Benedetti, di quegli atti di parola che nel pensiero pragmatico di J. L. Austin sono caratterizzati dalla loro intenzionalità e dal patto che stabiliscono col lettore. Nelle forze illocutorie identificate da Benedetti — "raccontare come ribellione," la "necessità del dire" e la "parola conquistata" (178-180) — si amplifica la natura rischiosa, urgente, e sovversiva di Gomorra come si rafforza la funzione controinformativa a cui l'opera aspira. La potenza della scrittura deriva, sicuramente, dall'offerta di un esempio concreto d'impegno e di resistenza, ma il suo vero potenziale si trova negli effetti modificanti che essa può avere nel territorio in cui prima si espande.

Alla voce denunciatrice, si lega una successiva caratteristica di Gomorra, da Benedetti individuata in "l'enunciato dell' intimità con il territorio" (178). Il senso d'appartenenza si manifesta nell'affetto per le persone e nella minuta conoscenza dei luoghi e della comunità civile così come nell'indignazione per lo sfruttamento di questi ambienti. A rendere inevitabile il viaggio nelle realtà e l'indagine sono, però, la presenza fisica e i legami essenzialmente sensibili con la realtà ritratta. Racconta Saviano che don Peppino, una volta finiti gli studi a Roma, era tornato a Napoli come "chi non riesce a togliersi di dosso un ricordo, un'abitudine, un odore" ( $G$ 242), e Saviano stesso ci si muove come "il cucciolo del Cane da caccia" nato con "l'odore di lepre nel naso" ( $G$ 308). Sia la conoscenza storica dei fatti presenti sia il netto rifiuto di assumere una posizione di neutralità o di distanza oggettiva distingue la sua prospettiva da quella dei giornalisti esterni: dalle faide camorriste essi raccolgono solo le superficialità più sensazionali, mentre l'autore 
autoctono vive le vicende da vicino e spesso nel loro svolgersi. Avendo frequentato spesso il mercato della droga di Secondigliano, egli è diventato noto nell'ambiente, ma poiché gli si attribuisce un valore "neutro, inutile" rispetto agli affari in corso, può seguire attentamente gli scambi e i movimenti, compresi quelli dei "visitors" chiamati a testare la merce prima che cominci a circolare $(G 75)$. Quanto sia fondamentale l'apparente neutralità, emerge in particolar modo nell'episodio dedicato ai ragazzi della Camorra. Tra una folla di curiosi raggruppatisi intorno al corpo ancor velato di una signora uccisa dai nemici del figlio, il narratore sente dei ragazzini che discutono di omicidi e, approfittando della loro conoscenza in materia, inizia a conversare con loro. Invitato dagli aspiranti camorristi si trova, poco dopo, nella pizzeria dove si riuniscono "i soldati" più giovani dello spaccio $(G$ 123). Tra la fame e l'effetto delle pasticche prese per reggere turni interminabili, sono ben disposti a sfogarsi e con il registratore in mezzo alla tavola, l'osservatore raccoglie i loro giudizi su un sistema sempre più redditizio ma che nella vita locale non lascia né prospettive né vie d'uscita. In casi come questi è essenziale "esserci perché le cose ti conoscano," perché tu diventi sufficientemente parte di una realtà non condivisa ma vissuta al punto da saperne riconoscere e denunciare meccanismi e nessi apparentemente inaccessibili, per poi offrire esempi e visioni di comportamenti e valori alternativi ( $G 83)$.

Per l'abilità d'osservare inosservato e immergersi in ambienti da cui rimane fondamentalmente estraneo, l'inchiesta di Saviano si avvicina alla ricerca antropologica e come essa segue l'obiettivo essenzialmente brechtiano di "pensare alle mani e ai piedi della storia" ( $G 234$ ). Ancor prima di queste scelte metodologiche, però, la determinazione di trasgredire le spietate leggi del territorio è motivata da un rifiuto assoluto dell'indifferenza e dei compromessi: una risolutezza che parte dall'essere e dalle percezioni per condurre verso il sapere e la ragione. Da lì giunge, infine, alla parressia - all'esercizio della libertà d'espressione a rischio della vita e con lo scopo di attivare un senso critico negli interlocutori. Considerando la fermezza manifestata in materia di questioni morali, il percorso liberatorio delineato in Gomorra rivela l'influenza della testimonianza poco riconciliante di Levi, ma si allinea ancor più visibilmente a quel documento intitolato "Per amore del mio popolo non tacerò" con cui don Peppino sfidò l'egemonia dei clan e il silenzio secolare che da sempre la circonda. Come quel testo religioso e programmaticamente civico, Gomorra comunica la determinazione di svolgere un ruolo chiarificatore e "azzannare la realtà, senza lasciarla se non dilaniandola" e si tratta in entrambi i casi di una scrittura concepita quale atto d'intransigenza ( $G 250)$. 
Indole suprema del cittadino libero, l'intransigenza è una virtù fondamentalmente controcorrente che si vede minacciata non solo in territori sommersi nell'omertà ma, come mostra Maurizio Viroli, in un'intera società dove la vita pubblica si è ridotta a una "corte" adulatoria e menzognera (La libertà 8). L'idea di un sistema composto, piramidalmente, di signori e sudditi si presenta in primo luogo come un'analisi del "potere enorme" di cui ha goduto Silvio Berlusconi, distribuendo favori e premiando i peggiori, ma le tesi avanzate riguardano più profondamente la perdita di quella "libertà morale" che incita i cittadini ad agire secondo coscienza a prescindere dagli interessi particolari e "dalle seduzioni della corte" (Viroli, La libertà 117). Una tale libertà prevede, innanzitutto, di votare alle elezioni e di difendere la patria da poteri antidemocratici; in questo senso, essere liberi non è tanto questione di avere certi diritti, quanto, piuttosto, di compiere i propri doveri ed esercitare i diritti civili. Chi volesse promuovere una rinascita civile dovrebbe, perciò, cominciare da fuori dalla corte e da principi fondamentalmente morali ancora prima che politici per dimostrare con le parole e i comportamenti che "l'intendimento non è realizzare un'altra corte ma costruire o ricostruire la libertà" (Viroli, La libertà 123).

È precisamente in vista dell'esigenza di rivalutare il concetto della libertà in termini illuministi che Roberta De Monticelli si sofferma sull'ideale di rendere ragione delle proprie azioni e di chiedere giustificazioni da chi amministra il potere. Fare fronte a diffusi mali sociali quali il familismo, il clientelismo e il particolarismo richiederebbe, innanzitutto, che la comune esperienza morale si riaprisse "alla relazione delle persone alla verità," riconoscendo che l'esercizio della libertà consiste nell'essere individualmente responsabile verso l'ethos collettivo (De Monticelli, 184-185). Davanti a tale quadro di pensieri critici si chiariscono ancor meglio la visione etica e il procedere inquisitivo di Gomorra: è per riprendere "la via di Socrate" che la narrazione parte dal panorama più esteso del capitalismo globale, identificandone l'origine nella corruzione, nella facile connivenza e nell'indifferenza, per poi denunciare la mancata giustificazione della realtà dei fatti: in tal modo si uniscono la disposizione universalmente umana di chiedere "perché" (Monticelli 186; 89-93) e la concezione dell'intransigenza quale "la determinazione di seguire il fine che la coscienza addita come giusto" (Viroli, Lintransigente 71). Va osservato che l'inflessibilità etica del narratore emerge non solo dalle domande poste nell'intento di svegliare chi per mancanza di alternative o per opportunismo non pone resistenza, ma anche nel veicolo con cui si rivolge alla civiltà contemporanea. Se la democrazia è indebolita, questo riflette secondo 
Viroli, anche la "distruzione della cultura scritta;" un'operazione sistematica, secondo lo studioso, che nella televisione trova il suo canale prediletto e il cui risultato effettivo è che il $6 \%$ degli italiani dichiarano di non sapere leggere ( $L a$ libertà dei servi 123-124). Contro la comunicazione superficiale e le intenzioni evasive di una cultura condiscendente e spesso degradante rispetto ai diritti e alla libertà civili, la scrittura di Saviano assume intonazioni inornate e suggestivamente dettagliate senza, appunto, escludere quello "slancio di poesia" richiesto perché la verità si trasmetta nelle sue forme più complesse (Saviano, "Gomorra, una storia").

Per chiedere ragione ai responsabili di tante vite sfruttate, danneggiate, ed eliminate, il narratore rintraccia, come abbiamo visto, i fondamenti di tanta distruzione, uno dei quali si nasconde nel cemento - il "petrolio del sud" ( $G$ 234). Non a caso è anche da quella fonte essenziale di viabilità per gli imperi della criminalità organizzata che si parte quando si tratta di difendere la dimostrabilità della conoscenza acquisita:

Io so e ho le prove. Io so come hanno origine le economie e dove prendono l'odore. L'odore dell'affermazione e della vittoria [...] E la verità della parola non fa prigionieri perché tutto divora e di tutto fa prova. E non deve trascinare controprove e imbastire istruttorie. Osserva, soppesa, guarda, ascolta. Sa [...] Le prove sono inconfutabili perché parziali riprese con le iridi, raccontate con le parole e temperate con le emozioni rimbalzanti su ferri e legni. Io vedo, trasento, guardo, parlo, e così testimonio, brutta parola che ancora può valere quando sussurra "È falso" all'orecchio di chi ascolta le cantilene a rima baciata dei meccanismi del potere. La verità è parziale, in fondo se fosse riducibile a formula oggettiva sarebbe chimica. Io so e ho le prove. E quindi racconto. Di queste realtà. (G 234)

Articolato sulle note del celebre articolo pasoliniano apparso sul Corriere della sera il 14 novembre 1974, la forza controinformativa del passo si costruisce con riferimento alla veridicità delle parole di chi racconta per rendere e chiedere ragione. Ciò che distingue il j'accuse anti-Camorra dal modello è l'enfasi sul valore verificante dei sensi: al contrario di Pasolini che sapeva per il merito d'essere un intellettuale ma senza possedere prove che rendessero verificabili le accuse rivolte al potere democristiano, Saviano ha visto, sentito e udito direttamente i fatti e con le prove derivate da queste scoperte può fare nomi e cognomi precisamente 
come fa quando cita rapporti giudiziari e ufficiali. In ultima istanza sembra infatti suggerire che non sarà l'inchiesta ma la testimonianza a trovare più ascolto, a meglio comunicare l'appello all'intransigenza e la tutela della giustizia, così come la conoscenza fondata sulla ragione potrà promuovere l'emancipazione dallo sfruttamento e dalle violenze di chi gestisce e nega la verità.

L'idea che la parola fondata sulle esperienze sensibili possa produrre prove in grado di definire l'impegno autoriale e civico del testimone evoca anche il valore documentale che essa acquisisce nell'atto d'iscrizione. Secondo Ferraris, ogni momento sociale e ogni atto fisico o di parola — "oggetti," questi, dal filosofo definiti "sociali" e perciò distinti da quelli "naturali" e aconcettuali — presenta un considerabile grado di "documentalità" giacché sono fissazioni di rapporti e trascrizioni di atti (Manifesto 76). Come dimostra la linguistica pragmatica, qualsiasi atto sociale può essere iscritto in un atto verbale, che si tratti di un'accusa, una ribellione o una manifestazione di autenticità e d'appartenenza. La documentabilità dei fatti e delle azioni indica innanzitutto che il mondo sociale possiede un distinto valore critico, ma suggerisce anche che la realtà del nostro tempo sia tutt'altro che fluida. Essa, al contrario, è sostenuta dagli stessi fondamenti a cui leghiamo le nostre esistenze ed esperienze morali, e le parole iscritte che ci si ancorano si rivelano "pietre [...] l'incubo del verba manent" (Ferraris, Manifesto 78). È precisamente la certezza che la realtà è in parte resistente e fermamente opposta a qualsiasi forma di manipolazione che ispira la decisione di esprimere una verità scandalosa, fondata non sul potere ma sul senso della giustizia, costruita non sull'iperreale né sulle falsificazioni, ma sui sensi e sulla ragione. Enunciata in un luogo spaziotemporale molto preciso e condivisa con lettori e ascoltatori, una tale scelta etica richiede un resoconto verificabile e innegabile. Che si tratti di un discorso politico, di un documento religioso o di una testimonianza giudiziaria, ci si trova comunque, secondo Ferraris, davanti a un oggetto sociale in quanto questi fenomeni registrati si legano al mondo sociale. Di conseguenza, essi costituirebbero anche atti d'iscrizione, giacché fissano questi stessi rapporti sociali in modo tale da avvicinarli alla dimensione dell'oggettività (Manifesto 75). La presenza fisica dell'autore campano tra persone e luoghi con cui s'identifica acquisisce, in questo senso, non solo un vantaggio metodico ma soprattutto un valore morale, giacché il testimone investe se stesso negli oggetti sociali trascritti così come nelle verità e nei valori enunciati affinché il testo raggiunga l'intento riformativo. Colta da questo punto di vista, la ragione si rivela come premessa di un impegno civile presentato ai lettori, come l'invito a un dialogo critico e continuo. $\grave{E}$, infine, non tanto a un potere enorme 
e indiscusso ma ai lettori e alla civiltà da loro rappresentata che l'autore chiede di rendere ragione ed è in base all'immediatezza stabilita tra esperienza, narrazione e scrittura che egli fa altrettanto, adoperando in tal modo un metodo capace di rivelare l'origine delle posizioni assunte e delle accuse pronunciate.

\section{Gonzaga University}

\section{Opere Citate}

Benedetti, Carla. "Roberto Saviano. Gomorra." Allegoria 57 (2008): 173-180. Brunkhorst, Hauke. Habermas. Trad. Leonardo Ceppa. Firenze: Firenze UP, 2008. Casadei, Alberto. “Gomorra e il naturalismo 2.0." Nuovi argomenti 45 (2009): 230-249.

De Caro, Mario, e Maurizio Ferraris. "Nuovo realismo e vecchia realtà." In Bentornata realtà. Il nuovo realismo in discussione. A cura di Mario De Caro e Maurizio Ferraris. Torino: Einaudi, 2012. v-xi.

De Monticelli, Roberta. La questione morale. Milano: Raffaello Cortina, 2010.

Donnarumma, Raffaele. "Nuovi realismi e persistenze: narratori italiani di oggi." Allegoria 57 (2008): 26-54.

Eco, Umberto. "Di un realismo negativo." In Bentornata realtà. Il nuovo realismo in discussione. A cura di Mario De Caro e Maurizio Ferraris. Torino: Einaudi, 2012. 93-112.

Ferraris, Maurizio. Manifesto del nuovo realismo. Roma-Bari: Laterza, 2012. . "Esistere è resistere." In Bentornata realtà. Il nuovo realismo in discussione. A cura di Mario De Caro e Maurizio Ferraris. Torino: Einaudi, 2012. 141-165.

. Realismo positivo. Torino: Rosenberg e Sellier, 2013.

Gallippi, Franco. "Roberto Saviano e 'la sfida del labirinto." In Negli archivi e per le strade: Il ritorno alla realtà nella narrativa d'inizio millennio. A cura di Luca Somigli. Roma: Aracne, 2013. 501-519.

Inglese, Andrea. "Immaginare il male in Roberto Saviano." In Italie en jaune et noir; la littérature policiére de 1900 à nos jours. Parigi: Presse Sarbonne nouvelle, 2010. 59-73. 
Kant, Immanuel. "Risposta alla domanda: Che cos'è illuminismo?” In Sette scritti politici liberi. A cura di Maria Chiara Pievatolo. Firenze: Firenze UP, 2011. 53-68.

Levi, Primo. Se questo è un uomo. Torino: Einaudi, 2005. . "Appendice a Se questo è un uomo." In Se questo è un uomo. Torino: Einaudi, 2005. 155-178. . I sommersi e i salvati. Torino: Einaudi, 2007.

Palumbo, Raffaello. "Narrazioni spurie: letteratura della realtà nell'Italia contemporanea." MLN 126. 2011: 200-223

Pocci, Luca. "Saviano e la new Italian po-ethic." In Negli archivi e per le strade: Il ritorno alla realtà nella narrativa d'inizio millennio. A cura di Luca Somigli. Roma: Aracne, 2013. 589-604.

Policastro, Gilda. "Roberto Saviano. Gomorra.” Allegoria 57 (2008): 185-190.

Ricciardi, Stefania. "Gomorra e l'estetica documentale nel nuovo millennio." Interférences littéraires/Literaire interferenties. Croisées de la fiction. Journalisme et littérature (2011): 167-186.

Saviano, Roberto. Gomorra. Viaggio nellimpero economico e nel sogno di dominio della camorra. Milano: Mondadori, 2006.

. "Saviano legge Primo Levi. Quel capolavoro che ha rischiato di non essere creduto." La Repubblica 6 novembre 2013. Web. 11/1/2017

. "Gomorra, una storia lunga dieci anni." La Repubblica 10 maggio 2016. Web. 11/2/2017.

Viroli, Maurizio. La libertà dei servi. Roma-Bari: Laterza, 2012. . Lintransigente. Roma-Bari: Laterza, 2012.

Wu Ming 1. "New Italian Epic versione 2.0. Memorandum 1993-2008: narrativa, sguardo obliquo, ritorno al futuro." Carmilla: letteratura, immaginario e cultura d'opposizione, 15 aprile 2008. Web. 11/2/2017. 
